\title{
DNA Repair Dysfunction in Pancreatic Cancer: A Clinically Relevant Subtype for Drug Development
}

\author{
Talia Golan, MD, a,b and Milind Javle, MDc
}

\begin{abstract}
Pancreatic ductal adenocarcinoma (PDAC) has a dismal prognosis, with a 5 -year survival rate of $\leq 7 \%$ across all stages. The limited success of conventional therapies for PDAC is at least partially attributable to its genetic heterogeneity. Precision targeting of known PDAC subtypes may positively affect the outcome of this disease. An important actionable subtype in this cancer is associated with DNA repair dysfunction, including cases with germline BRCA mutations. This subtype can be targeted by inhibitors of poly(ADP-ribose) polymerase (PARP). BRCA mutation-associated PDAC may be the first biomarker-driven subtype in this disease that can be successfully targeted. However, DNA repair defects can extend beyond the narrow spectrum of BRCA1/2 mutations in PDAC and are present in a large proportion of patients with familial PDAC. This review describes the subgroup of patients with PDAC with aberrant DNA repair and discusses diagnostic and therapeutic options.
\end{abstract}

J Natl Compr Canc Netw 2017;15(8):1063-1069 doi:10.6004/jnccn.2017.0133

Pancreatic ductal adenocarcinoma (PDAC) is a lifethreatening disease and is the fourth-leading cause of cancer death in the Western world. In 2016, an estimated 53,070 patients were diagnosed with PDAC and 41,780 people died of the disease in the United States. ${ }^{1}$ Most patients are diagnosed with advanced disease and median overall survival (OS) is $<6$ months, a figure that has not changed in the past 2 decades. ${ }^{2}$ Patients with PDAC with a good performance status experience a median OS of 9 to 11 months with combination therapies (gemcitabine/nab-paclitaxel or FOLFIRINOX). ${ }^{3,4}$ Despite numerous phase II/III studies of PDAC in the past decade, treatment options remain limited. ${ }^{5}$

Advances in other solid tumors have been possible due to biomarker selection of specific patient populations. Successful examples include ALK-positive lung cancer and HER2/neu-positive breast cancer. ${ }^{6,7}$ This review describes the subgroup of patients with DNA re-

\footnotetext{
From ${ }^{a}$ Department of Oncology, Sheba Medical Center, Tel HaShomer, and bSackler Faculty of Medicine, Tel Aviv University, Tel Aviv, Israel; and 'The University of Texas MD Anderson Cancer Center, Houston, Texas. Submitted March 20, 2017; accepted for publication July 6, 2017. The authors have disclosed that they have no financial interests,
}

pair mechanism aberrations, their identification, and therapeutic options.

\section{Historical Perspective}

A biological repair process follows chemical and environmental damage to DNA. The first link to defective DNA damage repair (DDR) and cancer arose from 2 clinical observations. Cleaver ${ }^{8}$ noted that patients with the autosomal recessive disorder xeroderma pigmentosum, who are at an increased risk of developing skin cancer, have difficulty in repairing UV light-related DNA damage. It was later demonstrated that the specific defect in this case resulted from mutations in the nucleotide excision repair system. The second observation in the 1990s was Lynch syndrome, a hereditary nonpolyposis colorectal cancer resulting from microsatellite instability (MSI), resulting from DNA mismatch repair impairment (MMR). ${ }^{9}$ Additionally, in 1991, King et $\mathrm{al}^{10}$ discovered that the BRCA1

arrangements, affiliations, or commercial interests with the manufacturers of any products discussed in this article or their competitors.

Correspondence: Talia Golan, MD, Oncology Institute, Sheba Medical Center, Tel HaShomer 52621 Israel.

E-mail: Talia.Golan@sheba.health.gov.il 
gene on chromosome 17 is involved in homologous recombination repair. Mutations in BRCA1/2 predispose women to familial breast and ovarian cancers, men to prostate cancer, and both sexes to upper gastrointestinal malignancies, including PDAC. Other cancers associated with defective DNA repair include Bloom syndrome, Werner syndrome, and Fanconi anemia. It has been hypothesized that cancers with a hereditary predisposition may frequently harbor mutations in DNA repair genes. ${ }^{11}$

\section{Germline DNA Repair Defects and PDAC}

The genetic basis of PDAC has been explored in several recent studies, and increased PDAC risk was found to be associated with inherited syndromes, of which the most intensely investigated group is with BRCA1/2 germline mutations. The prevalence of germline BRCA mutations was initially thought to be approximately $4 \%$ to $5 \%$ in PDAC. ${ }^{12}$ However, more recent and larger prescreening efforts for the POLO study (discussed later) demonstrated a higher prevalence, and has a prescreening selection for BRCA germline mutations. To date, $>1,000$ patients with metastatic PDAC who received platinumbased therapy have been screened, with an incidence rate of $6 \%$ to $7 \%$ detected in this unselected patient population. ${ }^{13}$ In high-risk populations, including the Ashkenazi Jewish population, the prevalence can be up to $20 \%{ }^{14,15}$ Other inherited PDAC syndromes have mutations in MMR genes, CDKN2A, and the hereditary pancreatitis genes. ${ }^{16,17}$

Next-generation sequencing studies have further noted PALB2 and ATM germline mutations in PDAC kindreds. In the PACGENE Consortium study, $10 \%$ of patients with PDAC and a positive family history (kindred with $\geq 2$ first-degree relatives with PDAC) had germline mutations in one of the genes mentioned..$^{18}$ The Ontario Cancer Registry used a more variable definition of family history and noted a lower incidence $(3.8 \%)$ of germline mutations in these genes using a 13-gene panel. ${ }^{19}$ Wider gene panels that incorporate additional DNA repair genes may be incremental in this regard. A recent 25-gene panel investigated 303 patients with familial PDAC and noted germline mutations in 10 genes (ATM, BRCA1, BRCA2, CDKN2A, PALB2, PMS2, BARD1, CHEK2, MUTYH, and NBN), most of which are involved in DNA repair, in $12 \%$ of the cases. ${ }^{20}$ Also of interest in this group were the Fanconi anemia (FANCC and FANCG) genes that are mutated (somatic or germline) in a fraction of young patients with PDAC. ${ }^{21}$ Uncommon pancreatic types, such as acinar carcinoma, have a higher prevalence of BRCA germline mutations. ${ }^{22}$ The role of several of these DNA repair genes has only recently been explored in PDAC.

\section{Somatic Mutations Associated With Aberrant DNA Repair}

With increased understanding of cancer genetics, the mechanisms of DNA repair have been increasingly understood over the past decade. It has been proposed that DDR defects also occur in sporadically occurring tumors, such as somatic BRCA1/2, ATM, RAD51, FANCC, and PALB2 mutations (Figure 1). The clinical implications of these mutations and response to DNA repair inhibitors are under investigation.

\section{DDR Dysfunction in Cancer}

Cancer cells typically have reduced or aberrant DNA repair and damage signaling response, and are reliant on alternative pathways that may be susceptible to DNA-damaging agents. Double-strand break repair (DSBR) pathways include nonhomologous end joining and homologous recombination repair, whereas single-strand breaks are repaired by base excision repair, MMR, and nucleotide excision repair. ${ }^{23}$ DDR is a well-defined process, and its components are classified into sensors, transducers, mediators, and effectors (Figure 2). ATM, ATR, and DNA-PK (DNAdependent serine/threonine protein kinase catalytic subunit) kinases are important components of this mechanism. ${ }^{24}$ Genetic sequencing has highlighted the importance of these pathways in PDAC.

Recent large-scale whole-genome sequencing (WGS) of PDAC has subtyped these cases based on molecular profiles (Table 1). These studies identified a well-defined subgroup of patients $(12 \%-17 \%)$ harboring DDR-deficient tumors. Bailey et $\mathrm{al}^{25}$ defined these patients as having an aberrant BRCA pathway. From a cohort of 456 tumors, 32 oncogenic genes were identified, which aggregated into 10 molecular mechanisms. The BRCA pathway included BRCA1/2, ATM, and PALB2, with a prevalence of $5 \%$ in germline and $12 \%$ in somatic PDACs. Waddell et $\mathrm{al}^{26}$ performed WGS studies of PDAC and classified this 


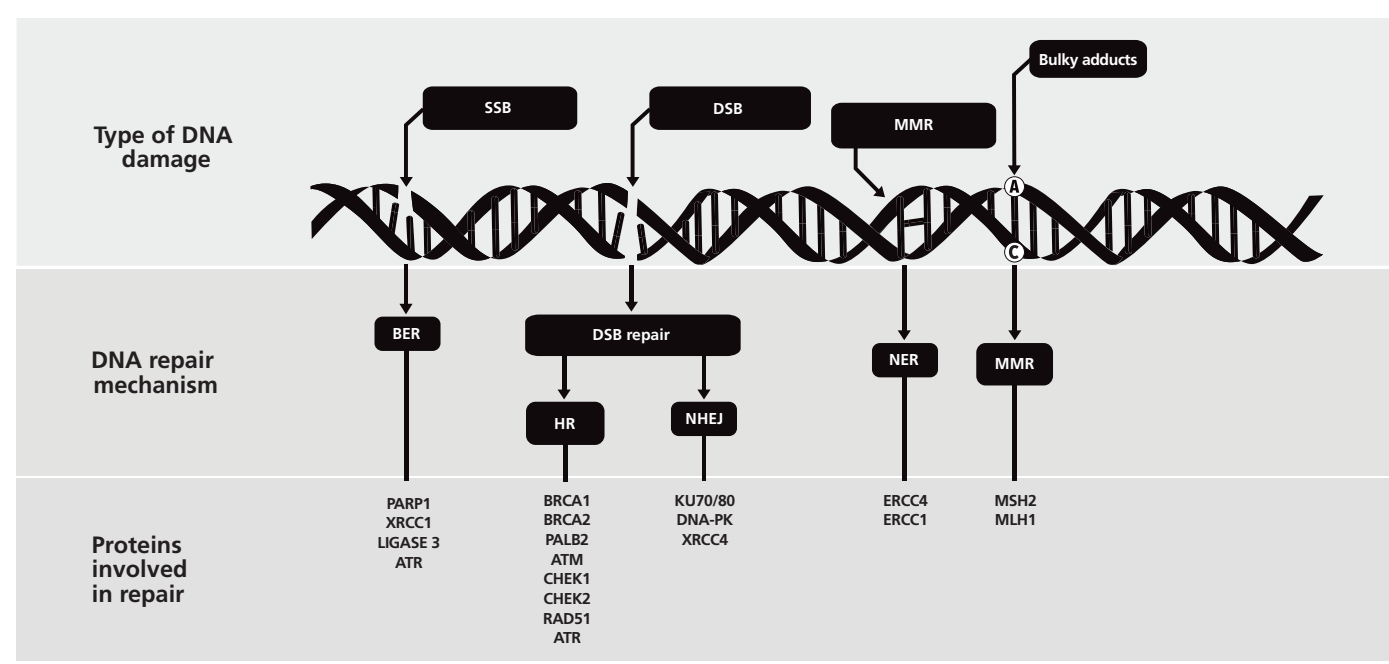

Figure 1. Types of DNA repair.

Abbreviations: BER, base excision repair; DSB, double-strand break; HR, homologous recombination repair; MMR, mismatch repair; NER, nucleotide excision repair; NHEJ, non-homologous end joining.

disease into 4 subtypes based on the patterns of structural variation: stable, locally rearranged, scattered, and unstable. Unstable genomes have $>200$ structural variations and are enriched with aberrant DNA repair genes, including BRCA1/2 and PALB2. Other genetic mutations detected in the unstable subtype include ATM, FANCM, XRCC4, and XRCC6 and a previously described BRCA mutationalsignature. $^{27}$ Connor et al $^{28}$ classified 255 PDACs based on mutation signatures and identified 4 major subtypes, including a DSBR group attributed to deficiencies in homologous recombination and DSBR that occurred in $11 \%$ of tumors.

Mutations in these genes, however, account for only half of the patients with unstable genomes or the DDR mutational signature. Additional mechanisms involved in aberrant DNA repair may include hypermethylation, which can silence the BRCA or PALB genes. Thus, the spectrum of DDR in PDAC is vast and still being understood. These studies also correlate well with clinical observations, in which there may be a clinical DDR phenotype (family history of BRCA-associated malignancies and responsiveness to platinum-based therapies without any known genomic DDR aberrations). ${ }^{29}$

\section{DDR Therapeutics}

Therapeutic strategies to target DNA repair pathways have been used in anticancer treatments for decades.
The best recognized among these are the chemotherapeutic DNA-damaging agents (platinum, topo II/I inhibitors, antimetabolites, alkylating agents, and radiation; Table 2). Novel DDR inhibitors can be categorized into 3 groups based on their mechanism of action: (1) agents targeting DDR sensor proteins, which include poly(ADP-ribose) polymerase (PARP) inhibitors; (2) agents targeting DDR-signaling proteins (DNA-PK, ATM, ATR); and (3) agents causing transient cell cycle delays, such as checkpoint inhibitors and WEE-1 inhibitors (Table 2). These agents are being investigated alone or in combination with DNA-damaging chemotherapy or radiotherapy. ${ }^{30,31}$

Foremost among the targeted DNA repair inhibitors in clinical development today are the PARP inhibitors. PARP has a role in DNA base excision repair and repair of DNA single-strand breaks. In patients with germline BRCA1/2 mutations, cancer cells depend on PARP-1 for their survival. In these cases, synthetic lethality induced by PARP-1 inhibitors has resulted in clinical benefit. ${ }^{32}$ The PARP-1 inhibitor olaparib demonstrated durable antitumor responses in BRCA1/2-mutated ovarian, breast, and prostate cancers. ${ }^{33-35}$ Olaparib and, more recently, rucaparib have been FDA-approved for BRCA1/2mutated ovarian cancer. Ongoing studies are investigating the role of PARP-1 inhibitors in PDAC with BRCA mutations and other DNA repair defects.

Single-agent PARP inhibitor therapy has demonstrated clinical responses in BRCA-associated 


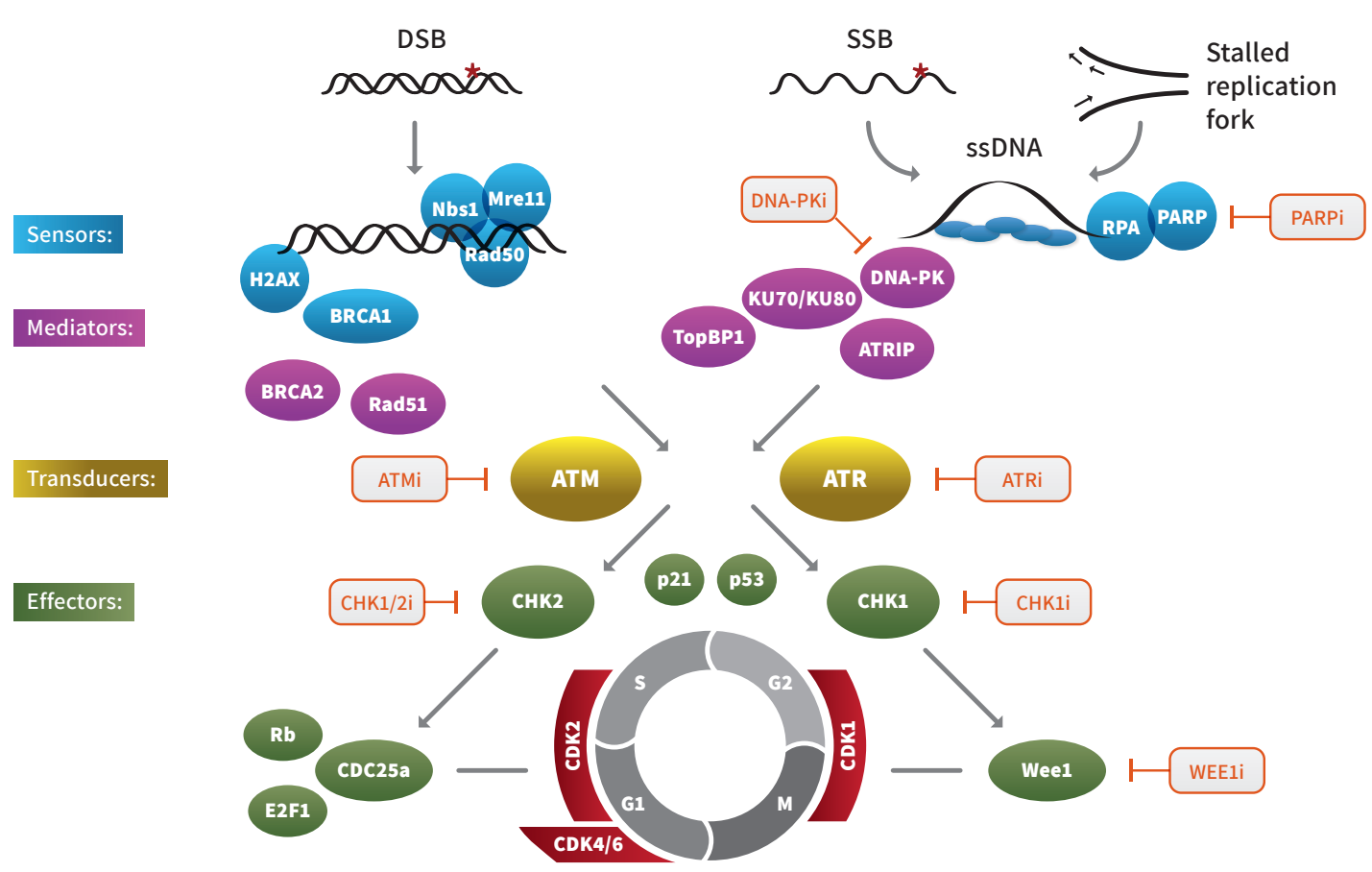

Figure 2. DNA repair pathway and drugs in development.

Abbreviations: DSB, double-strand break; SSB, single-strand break.

PDAC, and additional clinical trials evaluating PARP inhibitors in combination with platinumbased chemotherapy are ongoing. Two single-arm phase II studies of single-agent PARP inhibitors have been conducted in patients with germline BRCA-mutated malignancies. In one study, ${ }^{34} 23$ patients with germline BRCA mutation-associated PDAC were treated with olaparib after gemcitabine therapy; all patients had received prior gemcitabine and $>50 \%$ received a prior platinum-containing regimen. The objective response rate was $22 \%$, disease control rate was $57 \%$, progression-free survival

\begin{tabular}{|lll|}
\hline \multicolumn{2}{|c|}{$\begin{array}{l}\text { Table 1. DDR Subgroups in Pancreatic Ductal } \\
\text { Adenocarcinoma }\end{array}$} \\
\hline DDR Classification & Prevalence & Reference \\
\hline $\begin{array}{l}\text { Oncogenic alterations in } \\
\text { BRCA pathway genes }\end{array}$ & $\begin{array}{l}\text { Total: } 17 \% \\
5 \% \text { germline } \\
\end{array}$ & Bailey et a ${ }^{25}$ \\
\hline Unstable genome & $12 \%$ somatic & \\
\hline BRCA mutation signature & NA & Waddell et al ${ }^{26}$ \\
\hline Double-strand break & Total: $10.8 \%$ & Connor et al ${ }^{28}$ \\
deficiency & $4.4 \%$ germline & \\
& $1.6 \%$ somatic & \\
& $4.8 \%$ BRCAness & \\
\hline
\end{tabular}

Abbreviations: DDR, DNA damage repair; NA, not available. was 4.6 months, and OS was 9.8 months. Another study $^{36}$ investigated the role of rucaparib in 19 patients with PDAC who received 1 to 3 prior chemotherapy regimens for locally advanced or metastatic PDAC. Rucaparib demonstrated clinical benefit in 6 (32\%) of the 19 patients; 1 had a complete response and 2 had partial responses, whereas 4 had stable disease. The objective response rate, the primary end point for the study, was $16 \%$. No response was seen in platinum-refractory cases.

Emerging data from germline BRCA mutation-associated PDACs suggest that patients with platinum resistance may also be PARP inhibitorresistant. This cross-resistance was also observed previously in ovarian cancer. ${ }^{37}$ Therefore, it is suggested that although it is important to treat patients with DDR initially with platinum-based treatments for tumor burden reduction, there may be benefit to introducing PARP inhibitors into the therapeutic plan before platinum resistance develops. This strategy forms the background for the POLO study. In this international trial, patients with germline BRCA-mutant PDAC are randomly assigned after platinum-based induction therapy to maintenance 


\begin{tabular}{|lll|}
\hline Table 2. DDR Therapeutics \\
Mechanism & $\begin{array}{l}\text { Target } \\
\text { Inhibition }\end{array}$ & Compounds \\
\hline DDR sensors & PARP & $\begin{array}{l}\text { Niraparib, talazoparib, } \\
\text { olaparib, veliparib }\end{array}$ \\
\hline $\begin{array}{l}\text { DDR transducers/ } \\
\text { signaling pathway }\end{array}$ & DNA-PK & MSC2490484A, VX-984, CC-115 \\
ATM & AZD0156 \\
ATR effectors & CHK1 & $\begin{array}{l}\text { MK87767, LY2603618, } \\
\text { CCT245737, GDC0575 } \\
\text { LY2606368 }\end{array}$ \\
& CHK1/2 & AZD1775 \\
\hline $\begin{array}{l}\text { Binds base excision } \\
\text { repair sites }\end{array}$ & $\begin{array}{l}\text { Topo II } \\
\text { inhibitors }\end{array}$ & TRC102 \\
\hline
\end{tabular}

Abbreviations: DDR, DNA damage repair.

therapy with olaparib or to placebo (ClinicalTrials. gov identifier: NCT02184195).

\section{Germline DDR Dysfunction: Prognostic or Predictive}

PDACs with germline BRCA mutations are the most extensively investigated subgroup of the DDR dysfunction subgroup; these patients are identified through germline BRCA1/2 testing. Retrospective analyses indicated that these patients experience improved survival with platinum-based chemotherapy compared with non-platinum-based treatments or historic controls. ${ }^{38}$ The NCCN Clinical Practice Guidelines in Oncology (NCCN Guidelines) for Pancreatic Adenocarcinoma currently recommend treating these patients with platinum-based treatments, specifically gemcitabine/cisplatin. ${ }^{39}$ These observations have led to the hypothesis that " $B R$ CA-positivity" may be a predictive biomarker for platinum/PARP inhibitors. The prognostic role of BRCA mutations is less proven. In a retrospective case-control analysis of patients with surgically resected PDAC (stage I/II), no significant difference was demonstrated in median OS (37.06 vs 38.77 months; $P=.838$ ) or disease-free survival (14.3 vs 12.0 months; $P=.303$ ) between BRCA mutationpositive cases and controls. ${ }^{40}$ The prognosis of patients with surgically resectable BRCA-associated PDAC thus appears to be no different from that of patients with sporadic PDAC. The role of platinumbased adjuvant therapy in this setting is still unclear.

\section{Aberrant DNA Repair: Identification of Patients}

WGS studies were described earlier. The current clinical assays for DDR are limited to sequencing a panel of genes that are associated with an inherited risk of PDAC and other cancers. Given the large spectrum of DDR deficiency, functional assays will be the next frontier of research in this field.

\section{Functional Assays}

Focusing entirely on genomic alterations will not be sufficient due to hypermethylation and other epigenetic alterations of the DNA repair genes. Functional assays of DDR deficiency need to be explored in parallel. To obtain a comprehensive molecular understanding of homologous recombination repair process, rather than taking a single gene approach to analyze homologous recombination repair in cells, Peng et $\mathrm{al}^{41}$ used a genome-wide gene expression profiling approach to measure the cellular transcriptome reprogramming in homologous recombination-deficient (HRD) cells. They identified 230 genes that were differentially expressed between HRD cell lines (with BRCA1, RAD51, or BRIT1 loss) and controls $(P<.001)$, and designated this set the HRD gene signature. The HRD signature genes were involved in cell cycle regulation, DNA replication, and DNA repair pathways. The investigators showed responsiveness of this signature to PARP-1 inhibition. Additional assays include RAD51 foci at DNA damage sites in biopsies after neoadjuvant chemotherapy in breast cancer. Failure to generate RAD51 foci in cells was predicative of a pathologic complete response $(P=.011) .42$ Recently, deleterious ATM mutations have been recognized in the germline of families with familial PDAC, suggesting that ATM is a susceptibility gene in hereditary PDAC. ${ }^{18}$ Somatic ATM loss, as shown by immunohistochemistry (IHC), was observed in 50 (12.8\%) of 396 PDAC. ${ }^{43}$ Impaired ATM signaling may be identified by protein loss (IHC) and serve as a biomarker for the identification of the DDR signature in PDAC. PARP inhibition in combination with paclitaxel was recently evaluated in patients with gastric cancer harboring ATM loss; however, phase III results were negative. ${ }^{44}$

To summarize, the DDR phenotype has been identified in PDAC; however, the full spectrum is not well-defined. To further define the DDR pheno- 
type, we have initiated a clinical trial of PARP inhibition with olaparib in DDR-deficient PDAC. The patient population includes individuals with ATM loss, genetic aberrations known to lead to DDR dysfunction (other than BRCA mutations), and a history of $\geq 2$ breast, ovarian, or pancreatic cancers in $\geq 2$ first-degree relatives. All patients are being investigated for DDR signature, and the results will be correlated with radiologic response rate (ClinicalTrials. gov identifiers: NCT02677038 and NCT02511223).

\section{Immune Therapy and DNA Repair Deficiency}

In non-small cell lung cancer associated with high nonsynonymous mutation burden and DNA repair pathway mutations, improved rates of radiologic response and progression-free survival were demonstrated when treated with checkpoint inhibitors. ${ }^{45}$ BRCA1/2 mutated high-grade serous ovarian carcinoma is associated with a higher mutation burden compared with non-BRCA1/2-mutated tumors, higher predicted neoantigens, and higher expression of "immune" genes that are associated with cytotoxicity. ${ }^{46}$ IHC studies demonstrated that BRCA1/2mutated tumors exhibited significantly increased CD3-positive and CD8-positive tumor-infiltrating lymphocytes (TILs), as well as elevated expression of PD-1 and PD-L1 in tumor-associated immune cells compared with homologous recombination-proficient tumors. Preclinical efficacy was demonstrated in the combination of PARP inhibition with cytotoxic T-lymphocyte antigen-4 (CTLA-4) inhibitors in a BRCA1-deficient murine ovarian cancer model.
With this combination therapy, effector $\mathrm{T}$ cells were increased in the peritoneal cavity. ${ }^{47}$ Another DNA repair alteration associated with responsiveness to checkpoint blockage is defective MMR that results in MSI. Pembrolizumab is FDA-approved for MSIhigh cancers, including PDAC. ${ }^{48}$ MMR deficiency in PDAC, however, is rare.

In PDAC, concordance of PD-L1 expression with BRCA2 and BRCA1 mutations was demonstrated in $50 \%$ and $13 \%$ of cases, respectively. Compared with the general PDAC population, BRCA1-mutated cases have a higher incidence of PD-1 TILs, whereas BRCA2-mutated cases had a higher incidence of overexpressed PD-L1.49 WGS and transcriptome analysis identified $12 \%$ DDR cases in PDAC, and these were also associated with antitumor immune activation. ${ }^{28}$ DSBR and MMR subtypes were associated with increased expression of antitumor immunity, including activation of CD8-positive $\mathrm{T}$ lymphocytes and overexpression of regulatory molecules (CTLA-4, PD1 , and indolamine 2,3-dioxygenase 1), corresponding to a higher frequency of somatic mutations and tumor-specific neoantigens. ${ }^{28}$ Thus, considerable evidence currently exists to explore immune therapy approaches in DDR-deficient patients.

\section{Conclusions}

The DDR-deficient subtype of PDAC constitutes an important, clinically relevant, and actionable subset that extends beyond BRCA deficiency, and this subset has many targeted therapeutic possibilities. However, identification of this subset beyond the spectrum of BRCA1/2 deficiency remains a challenge.

\section{References}

1. Cancer Stat Fact: Pancreas Cancer. SEER Web site. Available at: https:// seer.cancer.gov/statfacts/html/pancreas.html. Accessed July 6, 2017.

2. Golan T, Sela T, Margalit O. et al. Short and long-term survival in metastatic pancreatic adenocarcinoma, 1993-2013 [abstract]. J Clin Oncol 2017;35(Suppl):Abstract 232

3. Conroy $\mathrm{T}$, Desseigne $\mathrm{F}$, Ychou M, et al. FOLFIRINOX versus gemcitabine for metastatic pancreatic cancer. N Engl J Med 2011;364:1817-1825.

4. Von Hoff DD, Ervin T, Arena FP, et al. Increased survival in pancreatic cancer with nab-paclitaxel plus gemcitabine. N Engl J Med 2013;369:16911703

5. Rahib L, Fleshman JM, Matrisian LM, Berlin JD. Evaluation of pancreatic cancer clinical trials and benchmarks for clinically meaningful future trials: a systematic review. JAMA Oncol 2016;2:1209-1216.

6. Shaw AT, Kim DW, Nakagawa K, et al. Crizotinib versus chemotherapy in advanced ALK-positive lung cancer. N Engl J Med 2013;368:2385-2394.

7. Di Leo A, Chan S, Paesmans M, et al. HER-2/neu as a predictive marker in a population of advanced breast cancer patients randomly treated either with single-agent doxorubicin or single-agent docetaxel. Breast Cancer Res Treat 2004;86:197-206.

8. Cleaver JE. Cancer in xeroderma pigmentosum and related disorders of DNA repair. Nat Rev Cancer 2005;5:564-573.

9. Lynch HT. Hereditary nonpolyposis colorectal cancer (HNPCC). Cytogenet Cell Genet 1999;86:130-135.

10. King MC. Localization of the early-onset breast cancer gene. Hosp Pract 1991;26:121-126.

11. Thompson LH, Schild D. Recombinational DNA repair and human disease. Mutat Res 2002;509:49-78.

12. Holter $S$, Borgida A, Dodd A, et al. Germline BRCA mutations in a large clinic-based cohort of patients with pancreatic adenocarcinoma. J Clin Oncol 2015;33:3124-3129.

13. Golan T, Oh DY, Reni M, et al. POLO: a randomized phase III trial of olaparib maintenance monotherapy in patients (pts) with metastatic pancreatic cancer (mPC) who have a germline BRCA1/2 mutation (gBRCAm) [abstract]. J Clin Oncol 2016;34(Suppl):Abstract TPS4152. 
14. Murphy KM, Brune KA, Griffin C, et al. Evaluation of candidate genes MAP2K4, MADH4, ACVR1B, and BRCA2 in familial pancreatic cancer: deleterious BRCA2 mutations in 17\%. Cancer Res 2002;62:3789-3793.

15. Stadler ZK, Salo-Mullen E, Patil SM, et al. Prevalence of BRCA1 and BRCA2 mutations in Ashkenazi Jewish families with breast and pancreatic cancer. Cancer 2012;118:493-499.

16. Salo-Mullen EE, O'Reilly EM, Kelsen DP, et al. Identification of germline genetic mutations in patients with pancreatic cancer. Cancer 2015;121:4382-4388.

17. Campa D, Pastore M, Gentiluomo M, et al. Functional single nucleotide polymorphisms within the cyclin-dependent kinase inhibitor $2 \mathrm{~A} / 2 \mathrm{~B}$ region affect pancreatic cancer risk. Oncotarget 2016;7:57011-57020.

18. Zhen DB, Rabe KG, Gallinger $S$, et al. BRCA1, BRCA2, PALB2, and CDKN2A mutations in familial pancreatic cancer: a PACGENE study. Genet Med 2015;17:569-577.

19. Grant RC, Selander I, Connor AA, et al. Prevalence of germline mutations in cancer predisposition genes in patients with pancreatic cancer. Gastroenterology 2015;148:556-564.

20. Petersen GM, Chaffee KG, McWilliams RR, et al. T. Genetic heterogeneity and survival among pancreatic adenocarcinoma (PDAC) patients with positive family history [abstract]. J Clin Oncol 2016;34(Suppl):Abstract 4108.

21. van der Heijden MS, Yeo CJ, Hruban RH, Kern SE. Fanconi anemia gene mutations in young-onset pancreatic cancer. Cancer Res 2003;63:25852588.

22. Lowery MA, Kelsen DP, Stadler ZK, et al. An emerging entity: pancreatic adenocarcinoma associated with a known BRCA mutation: clinical descriptors, treatment implications, and future directions. Oncologist 2011;16:1397-1402.

23. Jackson SP, Bartek J. The DNA-damage response in human biology and disease. Nature 2009;461:1071-1078.

24. Shiloh Y. ATM and related protein kinases: safeguarding genome integrity. Nat Rev Cancer 2003;3:155-168.

25. Bailey $\mathrm{P}$, Chang DK, Nones K, et al. Genomic analyses identify molecular subtypes of pancreatic cancer. Nature 2016;531:47-52.

26. Waddell N, Pajic M, Patch AM, et al. Whole genomes redefine the mutational landscape of pancreatic cancer. Nature 2015;518:495-501.

27. Alexandrov LB, Nik-Zainal S, Wedge DC, et al. Signatures of mutational processes in human cancer. Nature 2013;500:415-421.

28. Connor AA, Denroche RE, Jang GH, et al. Association of distinct mutational signatures with correlates of increased immune activity in pancreatic ductal adenocarcinoma. JAMA Oncol 2017;3:774-783.

29. Fogelman D, Sugar EA, Oliver G, et al. Family history as a marker of platinum sensitivity in pancreatic adenocarcinoma. Cancer Chemother Pharmacol 2015;76:489-498.

30. Abraham RT. Cell cycle checkpoint signaling through the ATM and ATR kinases. Genes Dev 2001;15:2177-2196.

31. Brown JS, O'Carrigan B, Jackson SP, Yap TA. Targeting DNA repair in cancer: beyond PARP inhibitors. Cancer Discov 2017;7:20-37.

32. Lord CJ, Ashworth A. BRCAness revisited. Nat Rev Cancer 2016;16:110 120 .

33. Ledermann JA, Harter P, Gourley C, et al. Overall survival in patients with platinum-sensitive recurrent serous ovarian cancer receiving olaparib maintenance monotherapy: an updated analysis from a randomised, placebo-controlled, double-blind, phase 2 trial. Lancet Oncol 2016;17:1579-1589.
34. Kaufman B, Shapira-Frommer R, Schmutzler RK, et al. Olaparib monotherapy in patients with advanced cancer and a germline BRCA1/2 mutation. J Clin Oncol 2015;33:244-250.

35. Mateo J, Carreira S, Sandhu S, et al. DNA-repair defects and olaparib in metastatic prostate cancer. N Engl J Med 2015;373:1697-1708.

36. Domchek SM, Hendifar AE, McWilliams RR et al. RUCAPANC: an open-label, phase 2 trial of the PARP inhibitor rucaparib in patients (pts) with pancreatic cancer (PC) and a known deleterious germline or somatic BRCA mutation [abstract]. J Clin Oncol 2016;34(Suppl):Abstract 4110.

37. Fong PC, Yap TA, Boss DS, et al. Poly(ADP)-ribose polymerase inhibition: frequent durable responses in BRCA carrier ovarian cancer correlating with platinum-free interval. J Clin Oncol 2010;28:2512-2519.

38. Golan T, Kanji ZS, Epelbaum R, et al. Overall survival and clinical characteristics of pancreatic cancer in BRCA mutation carriers. $\mathrm{Br}$ ] Cancer 2014;111:1132-1138.

39. Tempero MA, Malafa MP, Al-Hawary M, et al. NCCN Clinical Practice Guidelines in Oncology: Pancreatic Adenocarcinoma, Version 2.2016. Accessed July 7, 2017. To view the most recent version of these guidelines, visit NCCN.org.

40. Golan T, Sella T, O'Reilly EM, et al. Overall survival and clinical characteristics of BRCA mutation carriers with stage $\mathrm{I} / \mathrm{II}$ pancreatic cancer. Br J Cancer 2017;116:697-702.

41. Peng G, Chun-Jen Lin C, Mo W, et al. Genome-wide transcriptome profiling of homologous recombination DNA repair. Nat Commun 2014;5:3361.

42. Graeser M, McCarthy A, Lord CJ, et al. A marker of homologous recombination predicts pathologic complete response to neoadjuvant chemotherapy in primary breast cancer. Clin Cancer Res 2010;16:6159_ 6168.

43. Kim H, Saka B, Knight S, et al. Having pancreatic cancer with tumoral loss of ATM and normal TP53 protein expression is associated with a poorer prognosis. Clin Cancer Res 2014;20:1865-1872.

44. Bang Y, Boku N, Cjom K, et al. LBA25 - olaparib in combination with paclitaxel in patients with advanced gastric cancer who have progressed following first-line therapy: phase III GOLD study. Available at: http:// oncologypro.esmo.org/Meeting-Resources/ESMO-2016/Olaparib-incombination-with-paclitaxel-in-patients-with-advanced-gastric-cancerwho-have-progressed-following-first-line-therapy-Phase-III-GOLD-study. Accessed July 6, 2017.

45. Rizvi NA, Hellmann MD, Snyder A, et al. Cancer immunology. Mutational landscape determines sensitivity to PD-1 blockade in non-small cell lung cancer. Science 2015;348:124-128.

46. Strickland KC, Howitt BE, Shukla SA, et al. Association and prognostic significance of BRCA1/2-mutation status with neoantigen load, number of tumor-infiltrating lymphocytes and expression of PD-1/PD-L1 in high grade serous ovarian cancer. Oncotarget 2016;7:13587-13598.

47. Higuchi T, Flies DB, Marjon NA, et al. CTLA-4 blockade synergizes therapeutically with PARP inhibition in BRCA1-deficient ovarian cancer. Cancer Immunol Res 2015;3:1257-1268.

48. Le DT, Uram JN, Wang H, et al. PD-1 blockade in tumors with mismatchrepair deficiency. N Engl J Med 2015;372:2509-2520.

49. Sherri Z, Millis BL, Baker EH, et al. Multiplatform molecular profiling of pancreatic adenocarcinomas to identify BRCA1/2 mutations and PD-1/PD-L1 status with therapeutic implication [abstract]. J Clin Oncol 2015;33(Suppl):Abstract 4124 . 